This document was prepared in conjunction with work accomplished under Contract No. DE-AC09-96SR18500 with the U.S. Department of Energy.

This work was prepared under an agreement with and funded by the U.S. Government. Neither the U. S. Government or its employees, nor any of its contractors, subcontractors or their employees, makes any express or implied: 1 . warranty or assumes any legal liability for the accuracy, completeness, or for the use or results of such use of any information, product, or process disclosed; or 2 . representation that such use or results of such use would not infringe privately owned rights; or 3 . endorsement or recommendation of any specifically identified commercial product, process, or service. Any views and opinions of authors expressed in this work do not necessarily state or reflect those of the United States Government, or its contractors, or subcontractors. 


\title{
ENCAPSULATION OF PALLADIUM IN POROUS WALL HOLLOW GLASS MICROSPHERES
}

\author{
L. K. Heung, G. G. Wicks and R. F. Schumacher \\ Savannah River National Laboratory \\ Aiken, South Carolina, USA
}

\begin{abstract}
A new encapsulation method was investigated in an attempt to develop an improved palladium packing material for hydrogen isotope separation. Porous wall hollow glass microspheres (PWHGMs) were produced by using a flame former, heat treating and acid leaching. The PWHGMs were then filled with palladium salt using a soak-and-dry process. The palladium salt was reduced at high temperature to leave palladium inside the microspheres.
\end{abstract}

\section{INTRODUCTION}

Palladium absorbs all three hydrogen isotopes reversibly. But it absorbs protium more readily than deuterium, and deuterium than tritium. This is known as isotopic effect. This isotopic effect of palladium has been applied to separate hydrogen isotopes for many years ${ }^{1}$. In most applications the palladium is deposited on porous granules such as alumina and kieselguhr for support. The supported palladium called the packing material is packed in long columns which are used to separate the isotopes in ways similar to chromatography. The efficiency of such a column is highly dependent on the property of the packing material. Properties such as density and uniformity are critical. In an effort to produce a better packing material, porous wall hollow glass microcrospheres (PWHGMs) were produced and were filled with palladium. Palladium filled PWHGMs has the potential to be a better packing material for hydrogen isotope separation since it can have higher palladium density, improved uniformity and faster kinetics.

\section{EXPERIMENTAL METHOD}

The experimental work involved two parts. The first part was to produce porous wall hollow glass microspheres. The second part was to fill the PWHGMs with palladium.

Produce Porous Wall Hollow Glass Microspheres

Porous wall hollow glass microspheres were produced by a four-step process: 1) prepare glass particles of proper composition; 2) blow the glass particles over a propane flame to produce hollow glass microspheres (HGMs); 3) heat treat the HGMs to cause phase separation of the glass; 4) acid leaching to remove the soluble phase that leaves a porous wall. Individually, the method to produce HGMs and the method to produce porous glass have been known and commercially used for many years. For example, HGM has been produced by the $3 \mathrm{M}$ Company ${ }^{2}$ and the porous glass Vicor $^{\circledR}$ by Corning Glass Works ${ }^{3}$. However, combining these two processes to successfully produce PWHGMs is new.

Mixtures of glass frits consisting of approximately $60-90 \mathrm{wt} \% \mathrm{SiO}_{2}, 1-30 \mathrm{wt} \% \mathrm{~B}_{2} \mathrm{O}_{3}, 2-20 \mathrm{wt} \%$ alkali metal oxides, $0.005-0.5 \mathrm{wt} \%$ sulfur and the balance of various metal oxides were melted at about $1300{ }^{\circ} \mathrm{C}$ for 2 to 3 hours. After cooling to room temperature the melt was crushed in a plastic bag by hammer and ground to a fine powder using Tekmar ${ }^{\circledR}$ Grinders. The powder was screened by hand to less than 325 mesh ( $<45$ microns) before it was used as feed to produce HGMs in the flame former apparatus. 


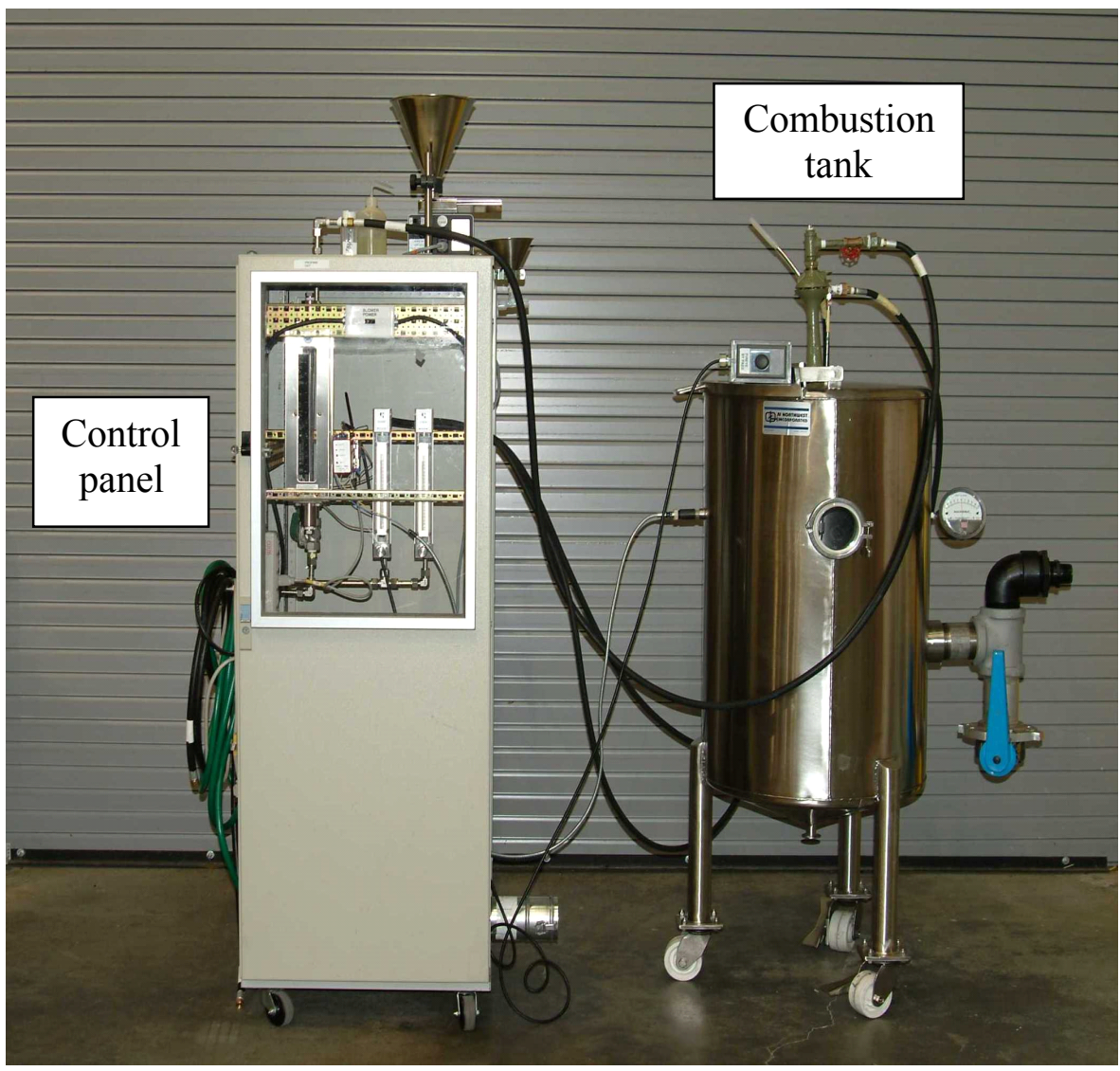

Figure 1. Flame former for producing hollow glass microspheres.

The flame former apparatus is shown in Figure 1. It consisted of a control panel and a combustion tank. The portable control panel housed the water, gas, and combustion controls. The movable stainless steel combustion tank contained the flame former, water sprays and a collection basin. The apparatus also included a portable propane tank and regulator. Propane and air at a ratio of about 1 to 25 were fed to a Hauck torch in the combustion tank. Glass particles in a Retsch vibratory powder feeder was carried to the flame with compressed air. Two water sprays set to supply a total of approximately 0.5 gallon per minute were used to quench the propane combustion flame to specific lengths, to act as a coolant for the stainless steel tank and to capture the HGMs and glass particles in the quench water. The quench water exited from the bottom of the stainless steel tank and was collected in plastic pails. The HGMs (density $<1.0 \mathrm{~g} / \mathrm{cc}$ ) floating on top of the pail (floaters) was collected with a vacuum collector and filtered out using No. 42 (6 micron) filter paper. The glass beads (density $>1.0 \mathrm{~g} / \mathrm{cc}$ ) and glass frit sunk to the bottom of the pail (sinkers) were collected separately.

The HGMs produced was heat treated at $620{ }^{\circ} \mathrm{C}$ for 18 hours before it was leached in 4 molar $\mathrm{HCl}$ solutions at $80{ }^{\circ} \mathrm{C}$ for 4 hours. Heat treatment caused phase separation which produced a silicarich phase and a more soluble sodium borate phase. Leaching removed the soluble sodium borate phase of the glass and created a porous wall. The porous wall HGMs sunk to the bottom of the solution. The sinkers were collected, water washed and dried at $100{ }^{\circ} \mathrm{C}$ overnight.

\section{Fill PWHGM With Palladium}

The porous wall hollow glass microspheres were filled with palladium by a soak-and-dry process and followed by hydrogen reduction. Saturated solutions of palladium salt were prepared at room temperature using de-ionized water. Palladium compounds that can be used include tetra amine, palladium nitrate $\left[\left(\mathrm{NH}_{3}\right)_{4} \mathrm{Pd}\left(\mathrm{NO}_{3}\right)_{2}\right]$ and palladium chloride $\left(\mathrm{PdCl}_{2}\right)$. Both can dissolve in water at 


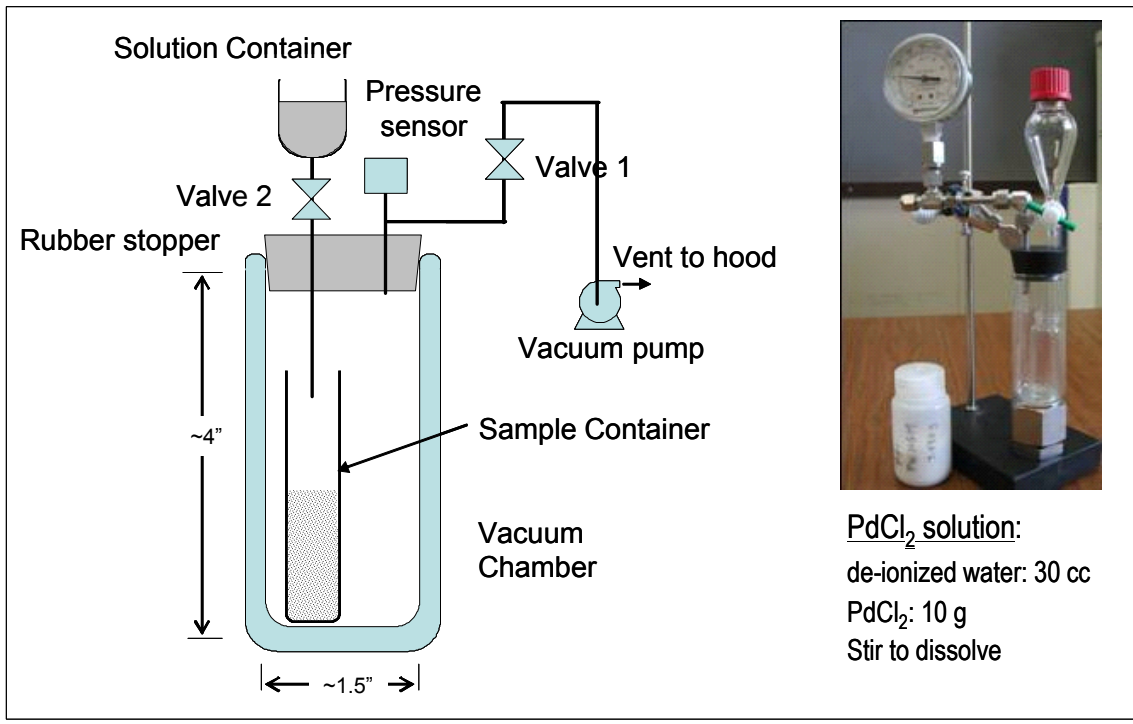

Figure 2. Apparatus for filling PWHGMs with palladium salt solution.

room temperature. At saturation, $\mathrm{PdCl}_{2}$ can dissolve up to about 2 mole per liter, $\left(\left(\mathrm{NH}_{3}\right)_{4} \mathrm{Pd}\left(\mathrm{NO}_{3}\right)_{2}\right.$ to about 1 mole per liter. The

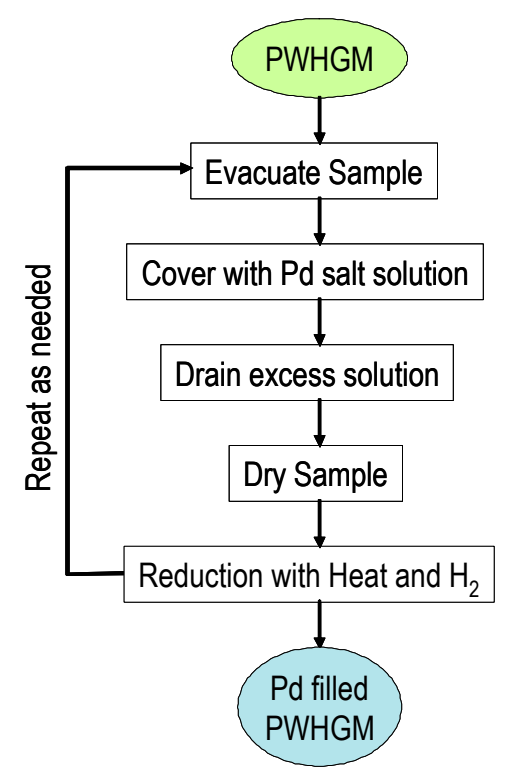

Figure 3. Steps to fill PWHGMs with palladium. palladium contents in 2 molar $\mathrm{PdCl}_{2}$ and 1 molar $\left(\left(\mathrm{NH}_{3}\right)_{4} \mathrm{Pd}\left(\mathrm{NO}_{3}\right)_{2}\right.$ are about $0.2 \mathrm{~g} / \mathrm{cc}$ and $0.1 \mathrm{~g} / \mathrm{cc}$, respectively. Palladium powder density can reach $4 \mathrm{~g} / \mathrm{cc}$ when packed tightly. This indicates that multiple fillings are required to reach high palladium density inside the microspheres.

In initial trials, sample of PWHGM was added to the solution under light mixing. The microspheres would initially float on top of the solution, but gradually sank as they were filled with the solution. Later it was found that under vacuum, the microspheres could be filled much more readily. To conduct vacuum fill, an apparatus shown in Figure 2 was used. The steps are shown in Figure 3. The vacuum chamber containing the PWHGM sample was first evacuated. The solution of palladium salt was added through a funnel to cover the sample. After the microspheres were soaked with the solution, excess solution was decanted and the sample was dried in open air or under vacuum. After the sample became dry, more solution were added. This soak-and-dry process was repeated 5 times to increase the content of palladium salt inside the microspheres. After the final drying, the salt filled sample was placed in a flowthrough container and was gradually heated to $450{ }^{\circ} \mathrm{C}$ under hydrogen purge for about 2 hours to reduce the palladium salt to pure palladium. A schematic of this high temperature reduction apparatus is shown in Figure 4.

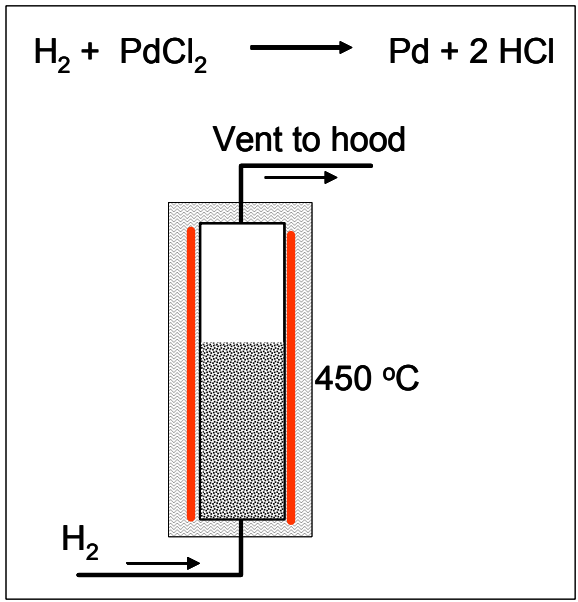

Figure 4. Palladium salt reduction with flowing hydrogen at high temperature.

\section{RESULTS AND DISCUSSIONS}

\section{Porous Wall Hollow Glass Microspheres (PWHGMs)}

The floaters collected in the flame forming step were hollow glass microspheres (HGMs). The size of the microspheres covered a range from about 5 to 150 microns with a true density of about 0.4 $\mathrm{g} / \mathrm{cc}$ measured with a helium pyknometer. The wall thickness was estimated to be between 1 to 5 microns. A SEM (scanning electron microscope) micrograph of the HGM is shown in Figure 5(I). The microspheres appear smooth and solid. When the same spheres were viewed under a light microscope 

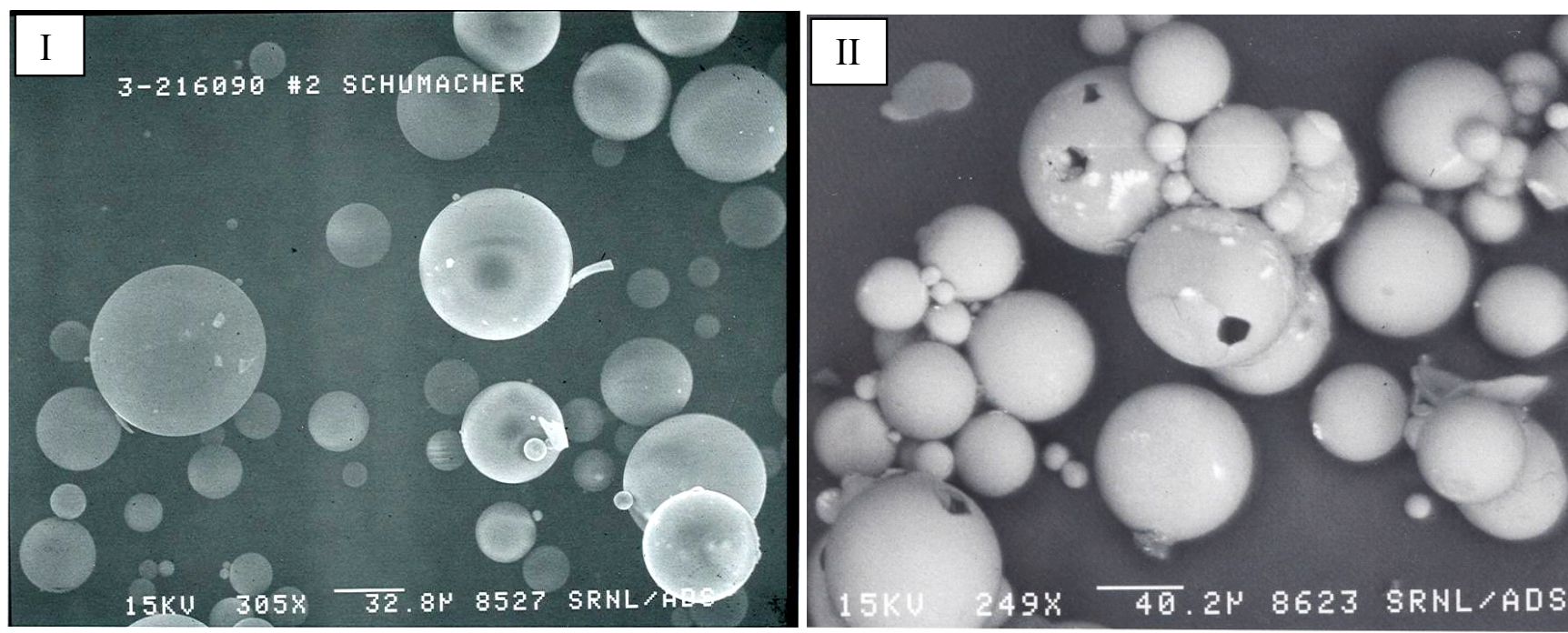

Figure 5. SEM micrographs of hollow glass microspheres (I) and porous wall hollow glass microspheres (II).

in transmission the HGMs appeared transparent. As the wall thickness becomes very thin the HGMs take on iridescence similar to a soap bubble.

The sinkers collected after the acid leaching were mostly PWHGMs besides a small amount of shards. A SEM micrograph of the PWHGMs is shown in Figure 5(II). Note that some of the larger microspheres were damaged with visible holes. It is known that large glass microspheres have thinner walls and are weaker than the small ones. The holes could have been created during leaching, washing or drying. Porosity analysis by the BET method using nitrogen and krypton showed that the surface area of the PWHGMs was approximately $30 \mathrm{~m}^{2} / \mathrm{g}$ which is a significant increase compared to HGM. The typical surface area of HGM is only about $1 \mathrm{~m}^{2} / \mathrm{g}$. The measured pore size covered a range between 300 to $1,000 \AA$ with an average of about $600 \AA$.

\section{Pd Filled PWHGMs}

Scanning Electron Microscope (SEM) examination was used to confirm that palladium did fill the inside of the microspheres. A sample of the filled and reduced microspheres was mounted on a sample stage and examined with the SEM. Micrographs were taken to record the appearances and locations of the individual microspheres. The same sample was then removed from the SEM and its microspheres were cracked open to reveal its interior before it was put back into the SEM for further examination. The cracking was done by pressing the mounted sample with a metal plate using the proper amount of force so that the mirospheres were opened but did not turn into debris. By this method, we were able to identify the individual microspheres before and after they were cracked open.
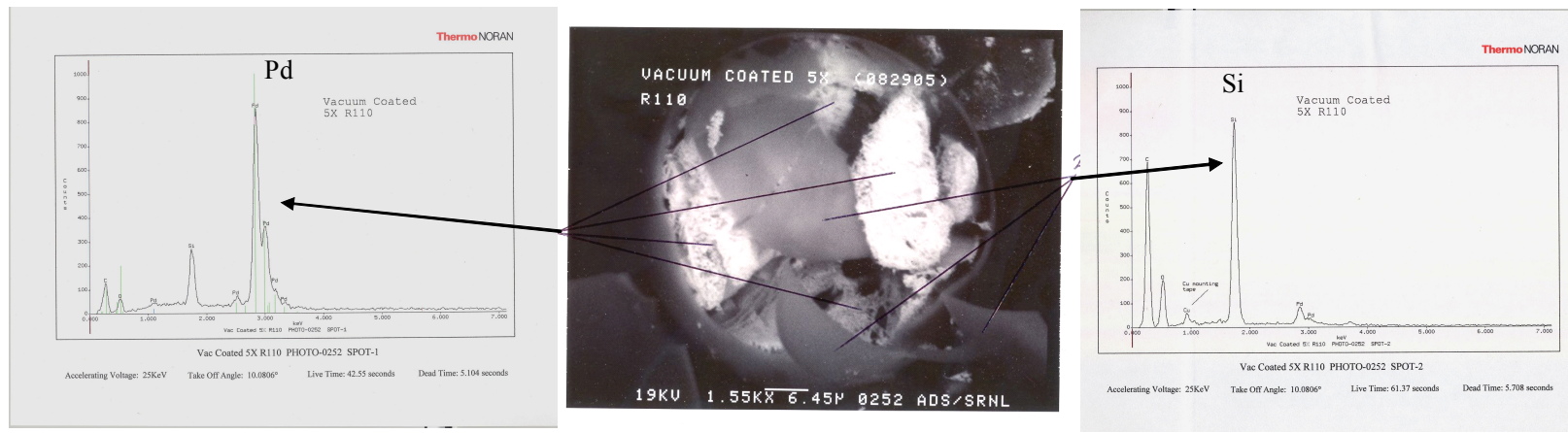

Figure 6. EDX spectra of a Pd filled PWHGM after smashed open to reveal its Pd. 
Figure 6 is a SEM micrograph of a smashed Pd filled PWHGM together with its two EDX (Energy Dispersed X-ray) spectra. In a SEM micrograph the palladium appeared white and the silica glass looked gray.

Figure 7 shows a particular mirosphere before and after it was cracked open. The palladium in this microsphere appears quite full, while its neighboring ones look mostly empty. The reason for this was not immediately obvious. A closer examination was necessary.

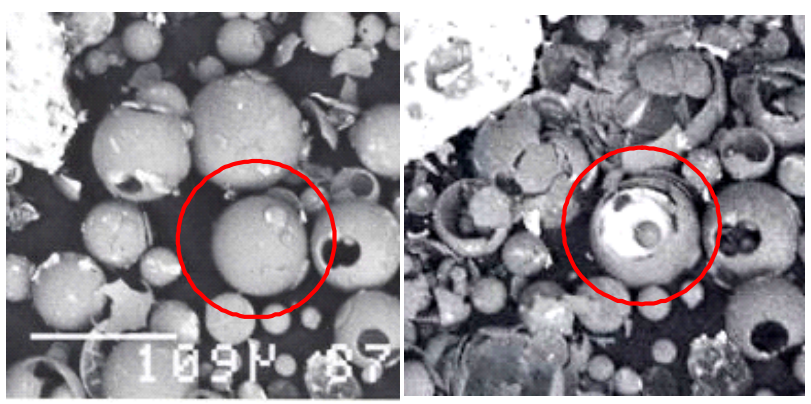

Figure 7. A PWHGM filled with Pd before and after it was cracked open to reveal the Pd.

In Figure 8, the micrographs of a group of microspheres before (I) and after (II) they were cracked open are compared side by side. The individual microspheres are identified by circles and letters. Note the ones labeled A, B, C and D. They appeared as good (no holes) microspheres in photo I. After cracked open in photo II they all revealed palladium (white) in their interiors. In contrast the ones labeled E, F and $\mathrm{G}$ which showed holes in photo I, did not contain any palladium in their interiors. This indicates that a microsphere must not have large holes in order to retain the Pd. The reason may be that the palladium salt solution can easily fill the microspheres with holes but can as easily flow out of them. And therefore little or no palladium left in the interior of these microspheres. Using similar reasoning, the pores of a microsphere must be sufficiently large for the solution to go in. Otherwise it cannot be filled.

Larger images of microsphere $\mathrm{E}$ in Figure 8 and the filled microsphere in Figure 7 are shown in Figure 9. The one on the left side (I) contains no Pd at all because it had a visible hole in its wall, while the one on the right (II) is filled nicely with Pd, presumably because it had the right porosity. The challenge for future work will be to produce a pure sample with all microspheres filled like the one shown in Figure 9(II).
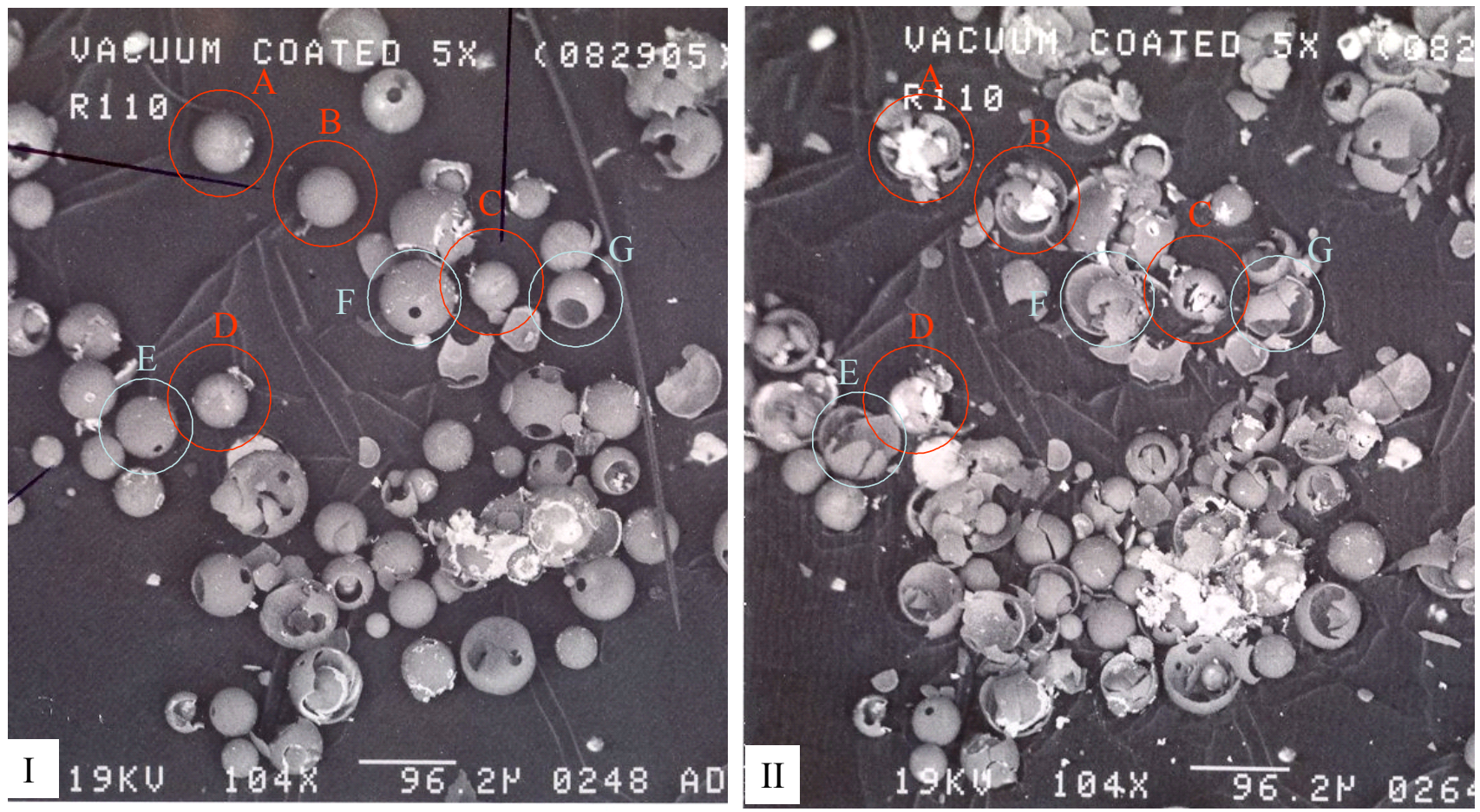

Figure 8. SEM micrographs of Pd filled PWHGM before (I) and after (II) they were cracked open. 


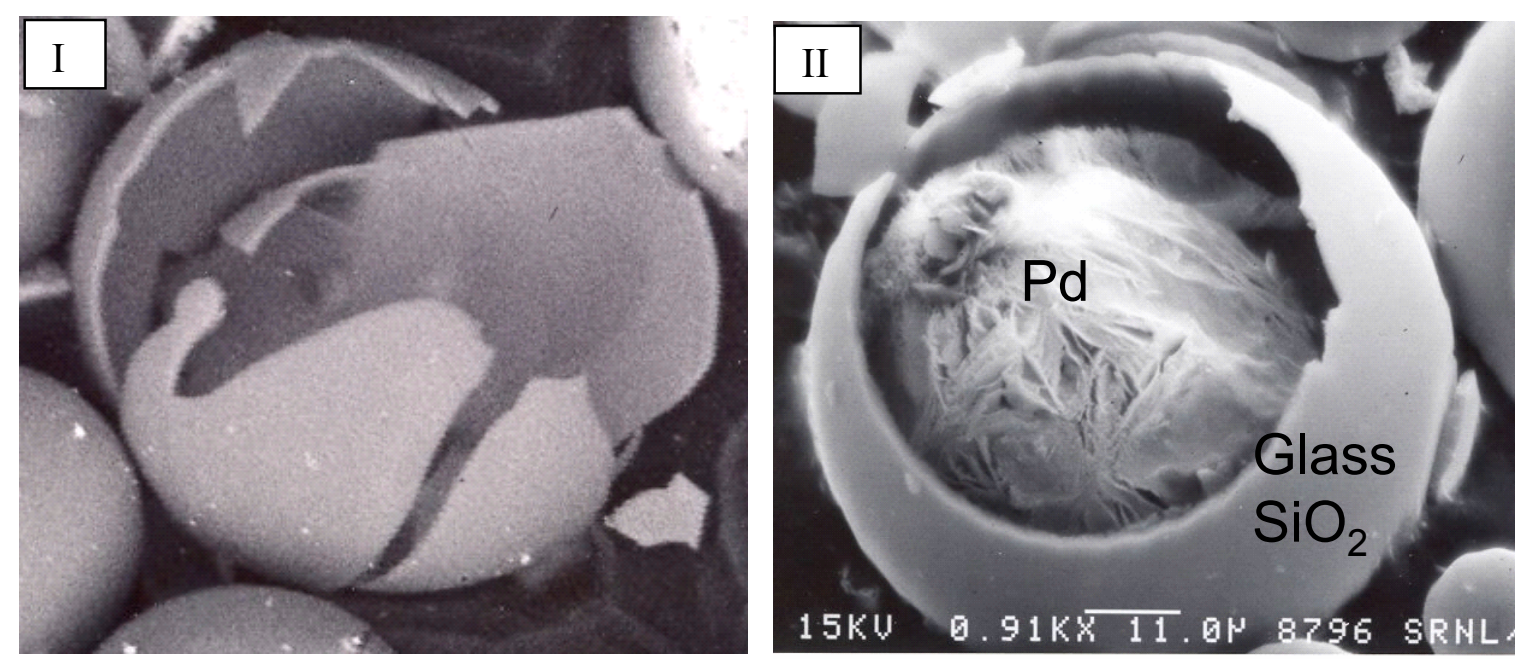

Figure 9. SEM micrographs comparing an empty and a filled PWHGM after cracked open.

\section{SUMMARY AND CONCLUSION}

We have successfully demonstrated a method to encapsulate palladium inside porous wall hollow glass microspheres. Hollow glass microspheres (HGMs) of proper composition were first produced using a flame former. The HGMs were then heat treated to cause phase separation to generate a silica rich phase and a soluble sodium borate phase. The soluble phase was removed by acid leaching that created porosity through the wall. The resulting porous wall hollow glass microspheres (PWHGMs) had diameter between 5 and 150 microns, wall thickness between 1 and 5 microns, surface area about $30 \mathrm{~m}^{2} / \mathrm{g}$ and pore size between 300 to $1,000 \AA$.

The PWHGs was filled with palladium salt using a soak-and-dry process. The palladium salt was then reduced at $450{ }^{\circ} \mathrm{C}$ with flowing hydrogen. The final product was Pd filled porous wall hollow glass microspheres.

The objective of this work was to investigate a new method to produce improved packing material for hydrogen isotope separation. But this technique can also be used to place other materials inside the microspheres for other applications. The wall of the filled microspheres can also be modified to different properties for different needs.

This work was exploratory in nature. It has demonstrated the feasibility of making and filling the microspheres. But more work is required to increase the understanding of the process and to improve the fabrication techniques for achieving homogeneous and consistent products.

\section{REFERENCES:}

${ }^{1}$ M. W. Lee, "Tritium separation using metal hydrides", 1986, http://sti.srs.gov/fulltest/dpms8611/dpms8611/pdf.

${ }^{2}$ Howell, P.A. (to 3M Co.), U.S. Patent 4,391,646 (July 5, 1983).

${ }^{3}$ Hood, H.P. \& Nordberg, (to Corning Glass Works), U.S. Patents 2,215,039 2,106,744 2,286,275 2,315,329 (1934 to 1943). 